\title{
10-W, 156-MHz All-fiber-integrated Er-Yb-doped Fiber Laser-amplifier System
}

\author{
Ihor Pavlov ${ }^{1}$, Emrah Ilbey ${ }^{1}$, Ebru Dülgergil ${ }^{2}$, F. Ömer Ilday ${ }^{1}$ \\ ${ }^{1}$ Department of Physics, Bilkent University, 06800 Ankara, Turkey \\ ${ }^{2}$ Meteksan Savunma, A.Ş., 06531, Ankara, Turkey \\ Email:pavlov@fen.bilkent.edu.tr
}

\begin{abstract}
We demonstrate all-fiber, high-power chirped-pulse-amplifier system, operating at $1550 \mathrm{~nm}$. $156-\mathrm{MHz}$ soliton oscillator seeds a two-stage single-mode amplifier with output power of $10 \mathrm{~W}$. After external compression, pulse duration is $0.6 \mathrm{ps}$.

(C)2011 Optical Society of America

OCIS codes: (140.3510) Lasers, fiber; (060.2320) Fiber optics amplifiers and oscillators; (320.7090) Ultrafast lasers.
\end{abstract}

In past decade, fiber lasers have emerged as one of the most rapidly developing branches of modern applied physics. There are several reasons for this, robust and free adjustment operation, high average power, which has exceeded multikilowatt level for cw operation and high beam quality [1]. Quick progress was driven also by a correspondingly impressive development of low-cost, high-brightness, fiber-coupled pump diodes as pump sources, enabling development of compact systems with extremely high efficiency [2]. Much of the activity in development of high-power fiber laser systems has concentrated on Yb-doped fibers, where normal material dispersion at this wavelength is advantageous in management of nonlinear effects for ultrashort pulses. There is demand for highpower, practical and low-cost Er-doped fiber laser systems, operating at $1550 \mathrm{~nm}$ wavelength. Operation at this wavelength is attractive for several reasons: The wavelength is in the eye-safe region and second-harmonic generation of $1550 \mathrm{~nm}$ is excellent for seeding Ti:sapphire amplifiers. There are also niche applications, such as ultrafast processing of silicon for photovoltaics, or corneal tissue surgery, where the high transparency of the material at $1.5 \mu \mathrm{m}$ is an advantage. Finally, compared to $1-\mu \mathrm{m}$ sources, it is better suited to seeding mid-IR parametric amplifiers and oscillators. However, to date, Er-doped fiber amplifier systems (EDFA) have lagged behind Yb-doped fiber amplifier systems (YDFA) in terms of efficiency and power. The main reasons include the much larger quantum defect for conventional $980 \mathrm{~nm}$ pumping, relatively smaller absorption cross-section, and impossibility to achieve high doping concentration [3], which, in turn, requires the use of long fibers, increasing nonlinear effects. One of the ways to avoid of foregoing problems is using an Er-Yb co-doping, which allows significantly increase of the pump absorption [1]. In this case, the pump energy is first absorbed by Yb ions, which is then transferred non-radiatively to the Er ions. The main drawback of this approach is the amplified spontaneous emission (ASE) generation at $1030 \mathrm{~nm}$ at high pump powers, which sharply decreases efficiency at $1550 \mathrm{~nm}$ and limits the output power level. $297 \mathrm{~W}$ of $\mathrm{CW}$ operation was demonstrated by using Er-Yb co-doped double-clad (DC) large mode area fiber [4]. In Ref. [5], the authors demonstrated an attractive possibility to reach about $10 \mathrm{~W}$ output power in single mode operation with a beam quality $\mathrm{M}^{2} \sim 1$. However, for ultrashort pulse amplification, Erfiber systems operating at 1550 is still not widely developed. In order to manage the nonlinear effects, which are strengthened by the anomalous dispersion of fibers at $1550 \mathrm{~nm}$, one approach is to use large mode area (LMA) fibers, in spite of the difficulty to maintain high beam quality [6-9]. In Ref. [9], the authors demonstrated amplification to an average power of $\sim 2.8 \mathrm{~W}$ at $15 \mathrm{MHz}$ repetition rate and $\mathrm{M}^{2}<1.1$ by using LMA fibers, which was pumped by a Raman fiber laser operating at $1480 \mathrm{~nm}$. High-energy amplification to the $\mu \mathrm{J}$-level has been demonstrated by several groups at this wavelength range at low average powers and with amplifier configurations, which are not all-fiber-integrated [10,11]. It is desirable to achieve higher powers and ideally with truly singlemode output, thus eliminating need for special fiber treatment.

Here, we report on the development of an all-fiber master-oscillator power-amplifier (MOPA) system, where the power amplifier is based on DC Er-Yb co-doped fiber. The system produces more than $10 \mathrm{~W}$ of average output power at $156 \mathrm{MHz}$ repetition rate. Despite the use of a quasi-singlemode gain fiber (core diameter of $10 \mu \mathrm{m}$ ), the amplifier output is through a fiber collimator comprised of pure singlemode fiber (Corning SMF-28) and absolutely no effort, such as fiber coiling, is applied to the gain fiber. This is the highest power truly singlemode operation of an ultrafast fiber amplifier at $1550 \mathrm{~nm}$, to the best of our knowledge. 
The general scheme of the experiment setup is shown in Fig. 1. As seed source, we use a passively modelocked Er-doped fiber laser, which operates in the soliton regime with a repetition rate of $156 \mathrm{MHz}$. The pulses are extracted through a 5\% fiber coupler; traverse an $80 \mathrm{~m}$-long segment of stretcher fiber with normal dispersion $(\mathrm{D}=$ $44 \mathrm{ps} / \mathrm{nm} . \mathrm{km}$ ). The stretched pulse duration is estimated to be $40 \mathrm{ps}$. After stretching, the signal is amplified in a 1.2 $\mathrm{m}$-long Er-fiber, which is core-pumped by a singlemode pump diode operating $976 \mathrm{~nm}$. The last stage of amplifier is a $6 \mathrm{~m}$-long DC Er-Yb-codoped fiber, which is backward-pumped into the cladding by two high power multimode diodes at $976 \mathrm{~nm}$ through a signal-pump combiner. The fibers comprising the signal-pump combiner and the output collimator are SMF-28 fiber, which is purely singlemode at $1.5 \mu \mathrm{m}$. The pulses from the amplifier were dechirped in a grating compressor with groove density of 600 lines $/ \mathrm{mm}$.

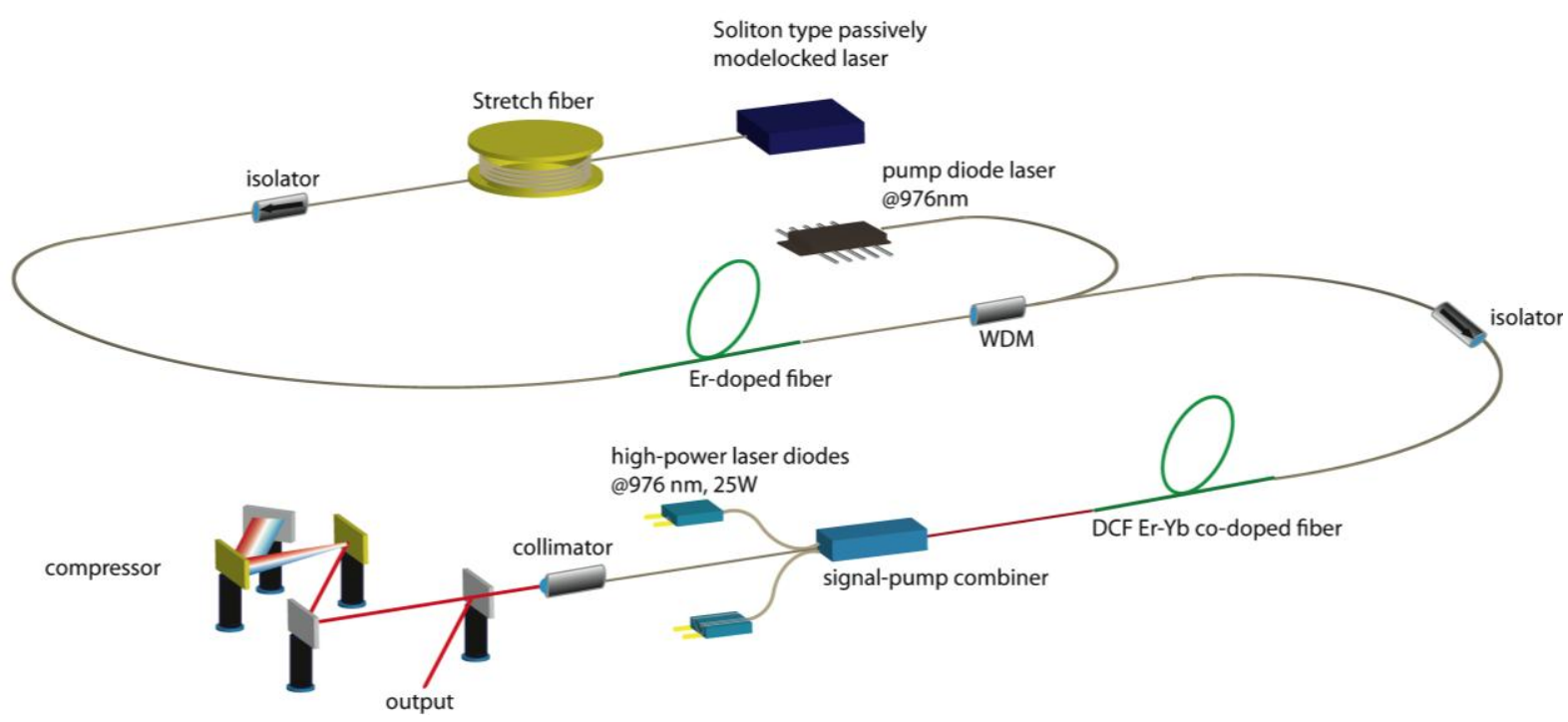

Fig. 1. Schematic of experiment setup.
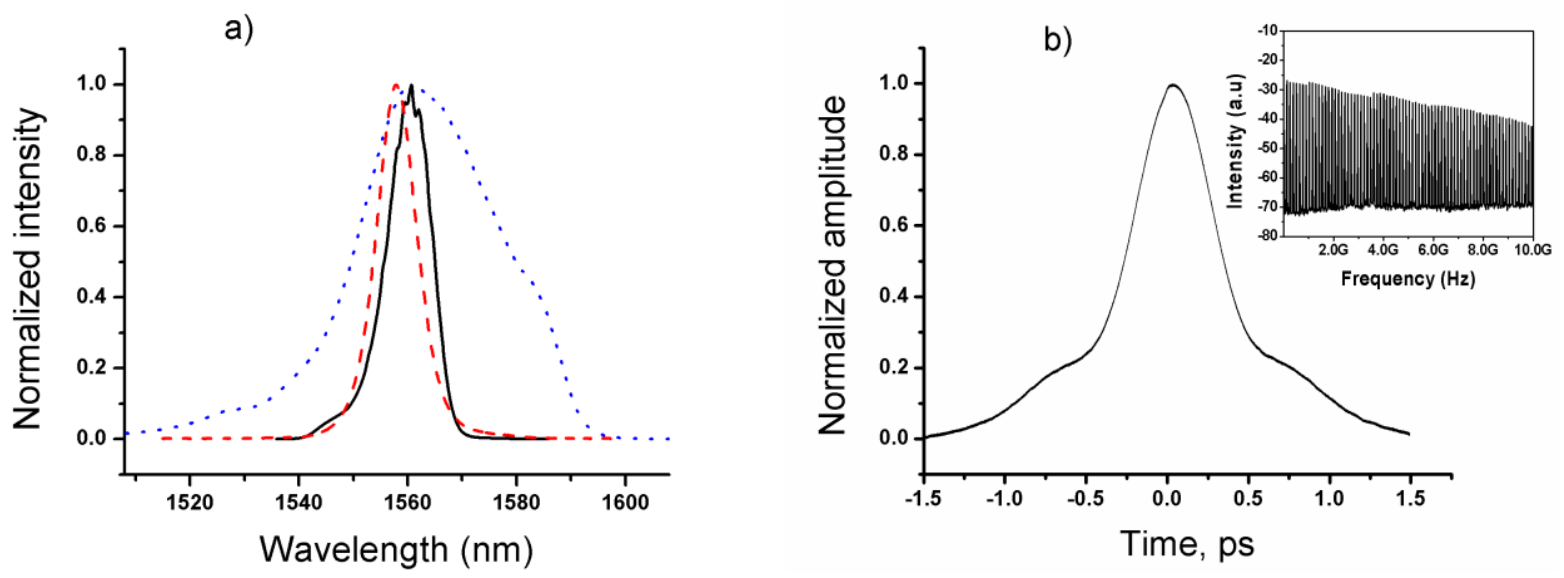

Fig. 2. (a) Measured optical spectra of the power amplifier at $10 \mathrm{~W}$ (black, solid line), preamplifier (dashed, red line) and seed oscillator (dotted, blue line). (b) Measured intensity autocorrelation of the pulses after compression. Inset: RF spectrum of the pulse train, indicating absence of double pulsing.

The average power of the seed pulses after the stretcher fiber is about $1 \mathrm{~mW}$, with corresponding energy of only $6.4 \mathrm{pJ}$. Thus, the preamplifier is used to boost the power up to $100 \mathrm{~mW}$. The optical efficiency is $30 \%$, but due to losses in the isolators and the WDM, the output power is reduced back to $\sim 80 \mathrm{~mW}$. The slope efficiency of the high-power amplifier was around $\sim 40 \%$ upon first construction, but it was reduced to $\sim 36 \%$ after several tens hours 
of operation. Several different versions of the amplifier were constructed. We have found that this reduction depends on the quality of the splice between gain fiber and passive fiber of combiner as well as on the quality of the cooling system of the gain fiber, especially near the splice point. Therefore, we believe the deterioration is at least partially due to degradation of the coating and is not caused entirely by the usual process of photodarkening. However, after this reduction, the amplifier stabilizes at $10.2 \mathrm{~W}$ of power and appears to be completely stable. We believe the output power can be increased further, but in our experiments, we are limited by the power handling capability of the signal-pump combiner, which has two pump ports with a maximum specified power of $\sim 15 \mathrm{~W}$ per port. The output spectra recorded for the seed pulses, after preamplification and after power amplification are shown in Fig. 2(a). The total amplification factor of the system is more than $40 \mathrm{~dB}$, including the losses. As a result, we observe significant gain narrowing. Unlike previous works [5], at this pump level, we do not observe any spurious ASE at $1030 \mathrm{~nm}$ neither for backward pumping nor for forward pumping, which was also tried. The pulses are checked against multiple pulsing using a high-speed photodetector and an RF spectrum analyzer. After dechirping the pulses in a standard grating compressor, the pulses are characterized via intensity autocorrelation (Fig. 2(b)). The full-width at half-maximum pulse duration is inferred to be $\sim 600 \mathrm{fs}$, assuming a Gaussian pulse shape. The pulse width is nearly twice the transform-limited pulse duration, which is limited by the onset of the self-phase modulation in the amplifier, as well as mismatched third-order dispersion of the fiber stretcher and the grating compressor.

In conclusion, we have demonstrated an all-fiber-integrated oscillator-amplifier system comprising of a 150$\mathrm{MHz}$ oscillator operating in the soliton regime and seeding a fiber amplifier. Beam propagation everywhere in the system is in optical fiber and fiberized components until the grating compressor. As such, the system is misalignment free. The amplifier generates $10 \mathrm{~W}$ of average power at the collimator output, which is made of pure SMF-28 fiber. Therefore, the output is truly single-mode and diffraction limited. After dechirping, the pulse duration is $0.6 \mathrm{ps}$. We expect this robust system to be of use in various applications requiring high-power, highrepetition-rate pulses at an eye-safe wavelength.

\section{References}

[1] D. J. Richardson, J. Nilsson, and W. A. Clarkson, "High power fiber lasers: current status and future perspectives [Invited],” J. Opt. Soc. Am. B 27, B63-B92 (2010).

[2] J. Price, et al., "High brightness fiber coupled pump laser development," retrieved from http://www.nlight.net/nlight files/file/technical_papers/PW10/Jan\%2030\%20High\%20Brightness\%20Fiber\%20Coupled\%20Pump\%20Lasers.pdf (2010).

[3] O. Lumholt, T. Rasrnussen, and A. Bjarklev, "Modelling of extremely high concentration erbium-doped silica waveguides," Elec. Lett., 29 495 (1993).

[4] Y. Jeong Y, et al., "Erbium:Ytterbium co-doped large core fiber laser with 297-W continuous-wave output power," IEEE J. Sel. Top. Quant. 13,573 (2007).

[5] B. Morasse, "10W ASE free single mode high power double cladding $\mathrm{Er}^{3+}-\mathrm{Yb}^{3+}$ amplifier," retrieved from http://www.coractive.com/pdf/publications/Article_10W_Er_Yb\%20PW2007.pdf (2010).

[6] J. C. Jasapara, M. J. Andrejco, J. W. Nicholson, A. D. Yablon, and Z. Várallyay, "Simultaneous direct amplification and compression of picosecond pulses to 65-kW peak power without pulse break-up in erbium fiber," Opt. Express 15, 17494 (2007).

[7] J. C. Jasapara, A. DeSantolo, J. W. Nicholson, A. D. Yablon, and Z. Várallyay, "Diffraction limited amplification of picosecond pulses in $1170 \mu \mathrm{m}^{2}$ effective area erbium fiber," Opt. Express 16, 18869 (2008).

[8] J. C. Jasapara, M. J. Andrejco, A. D. Yablon, J. W. Nicholson, C. Headley, and D. DiGiovanni, "Picosecond pulse amplification in a corepumped large-mode-area erbium fiber," Opt. Lett. 32, 2429 (2007).

[9] J. C. Jasapara, et al., "Diffraction limited fundamental mode operation of core-pumped very-large-mode-area Er fiber amplifiers," IEEE J. Sel. Top. Quant. 15, 3 (2009).

[10] M. E. Fermann, A. Galvanauskas, M. Hofer, "Ultrafast pulse sources based on multi-mode optical fibers,” Appl. Phys. B 70, S13 (2000).

[11] F. Morin, F. Druon, M. Hanna, and P. Georges, "Microjoule femtosecond fiber laser at $1.6 \mu \mathrm{m}$ for corneal surgery applications," Opt. Lett. 34, 1991-1993 (2009) 